\title{
The Design and Realization of Intelligent Test Paper and Achievement Analysis System for Financial Accounting Informatization in Higher Vocational Colleges
}

\author{
Liuwei Chen \\ Hubei Three Gorges Polytechnic, Hubei, Yichang, 443000, China \\ 390082@qq.com
}

Keywords: Higher vocational colleges, The group, Performance analysis, Design and implementation, Accounting informatization

\begin{abstract}
With the rapid development of the computer era, more and more colleges and universities use computers to assist the teaching process and manage the question bank through computers. The use of computer technology can effectively, scientifically and systematically improve the management of question bank, at the same time can also assist the teaching work, which will be conducive to improve the teaching quality and students' learning effect, so as to realize the separation of the evaluation department and the education part of the university management. This paper is mainly aimed at the higher vocational colleges through the computer intelligence to carry on the organization of the paper and analysis of the performance of the defects to carry on the analysis, the use of the computer random algorithm to take the initiative to achieve the organization of the paper function, so as to improve the higher vocational colleges intelligence organization of the paper and performance analysis of the management system.
\end{abstract}

\section{Introduction}

\subsection{Measurement Standards for the Quality of Financial Examination Papers}

The reliability of the financial examination paper can also be called the reliability of the financial examination paper. The reliability of the financial examination paper should include two aspects, namely the reliability of the examination paper itself and the reliability of the grading. The reliability of scoring is mainly judged by whether the scoring standard is accurate or not. The specific formula is as follows:

$$
R=\frac{\sum_{t=1}^{n}\left(M_{1}-\bar{M}\right)\left(N_{1}-\bar{N}\right)}{\sqrt{\sum_{t=1}^{n}\left(M_{1}-\bar{M}\right)^{2} \cdot \sum_{i=1}^{n}\left(N_{1}-\bar{N}\right)^{2}}}
$$

The validity of the financial examination paper is the main way to measure whether the examination paper is tested or not. The contents of the examination paper should be consistent with the teaching syllabus. Only through the validity of the financial examination paper can students accurately master the level of financial knowledge they learn.

$$
r=\frac{\sum_{i=1}^{n}\left(M_{1}-\bar{M}\right)\left(N_{1}-\bar{N}\right)}{\sqrt{\sum_{i=1}^{n}\left(M_{1}-\bar{M}\right)^{2} \cdot\left(N_{1}-\bar{N}\right)}}
$$

\subsection{Basic Attribute Indexes of the Test Bank}

The common test question type in examination paper basically has: multiple choice question, multiple choice topic, judgment question, fill in the blank question, calculate a question, discuss a question, short answer a question and so on, the knowledge point on examination paper is to the teaching outline or the knowledge point in the examination outline specially refers to carry on a roll, in a roll is, will carry on a roll according to the knowledge point completely. At last, the difficulty in 
the paper is divided into five aspects: simple, easy, general, difficult and difficult. The difficulty in the paper is an important standard to measure the quality of the paper, which is generally expressed as a percentage. When the computer selects the examination questions, the examination papers of finance discipline will be selected according to different test methods, purposes and stages, and the classification details of the difficulty are shown in table 1.

Table 1 Grade of Difficulty for Test Questions

\begin{tabular}{|l|l|l|l|l|l|}
\hline Grade of difficulty & Easy & More easily & Medium & Harder & Difficult \\
\hline Difficulty value & {$[0,0.2]$} & $(0.2,0.4]$ & $(0.4,0.6]$ & $(0.6,0.8]$ & $(0.8,1]$ \\
\hline
\end{tabular}

Calculation formula of difficulty value $\mathrm{P}$ of objective problem is:

$$
p=1-\frac{R}{N}
$$

Let $\mathrm{N}$ be the total number of people on the test, and $\mathrm{R}$ be the number of people who got the question right. With the above formula, you can see how difficult the test is. If $\mathrm{p}=0$, it means that all the answers are correct and the difficulty of the test is relatively low. If $p=1$, it means that no one answered the question correctly and the difficulty value is high. The formula for calculating the difficulty value $\mathrm{P}$ 'of the subjective question is:

$$
p^{\prime}=\frac{\bar{X}}{K}
$$

The degree of difficulty of each examination paper can be used to judge the difficulty of the examination paper of finance subject. The specific calculation formula is as follows:

$$
P=\frac{\sum_{i=1}^{n} P_{i} K_{i}}{\sum_{i=1}^{n} K_{i}}
$$

Differentiation is to point to by financial examination paper can distinguish between the levels of different people, this is the main measure of quality policy, is by calculating the unit volume when the main basis of topics, by the degree of differentiation in the financial questions can differentiate between different levels of the participants, the greater the using value of the sets of the examination, the distinguish formula is as follows:

$D=P H-P L$

The following figure shows the cognitive level by distinguishing degree, and the specific cognitive level communication is shown in figure 1.
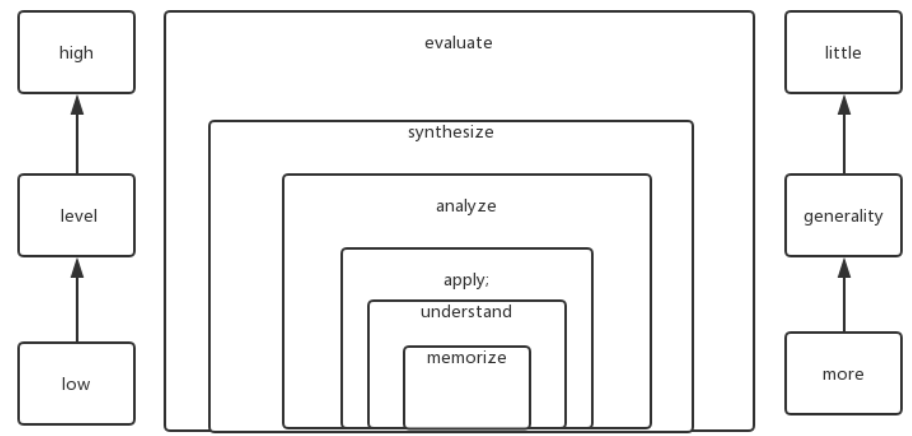

Fig.1 The Cognitive Level By Distinguishing Degree and The Specific Cognitive Level Communication

Table 2 Classification and Hierarchy of Educational Objectives in the Cognitive Field, the Specific Differentiation Data Evaluation Table is Shown in Table 2. 
Table 2 Grading Ratings For Test Questions

\begin{tabular}{|l|l|}
\hline Discrimination D & evaluate \\
\hline$\geqq 0.40$ & tall \\
\hline $0.30 \sim 0.39$ & Higher, can make improvement \\
\hline $0.20 \sim 0.29$ & Available and needs to be modified \\
\hline$\leqq 0.19$ & Poor, must eliminate or revise \\
\hline
\end{tabular}

\section{System Design}

\subsection{Overall Design}

Through C\# design to achieve higher vocational colleges computer intelligence group paper and performance analysis system, a total of three levels. First, the WEB layer is used to realize the service and human-machine interaction. The WEB layer can also be called the form layer. The ASPX page is used to complete the simple form control distribution, and the business logic layer is perfectly verified. Second, the business logic layer is mainly responsible for the management of efficient question bank resources. Users need to use the WEB layer for client-side operations. After the business logic layer receives the data, it will pass the processing results to the WEB layer and show the data to users through the WEB layer. Third, the data access layer mainly accesses the data information in the database to ensure the standardized and efficient system management data. See figure 2 for the specific model diagram.

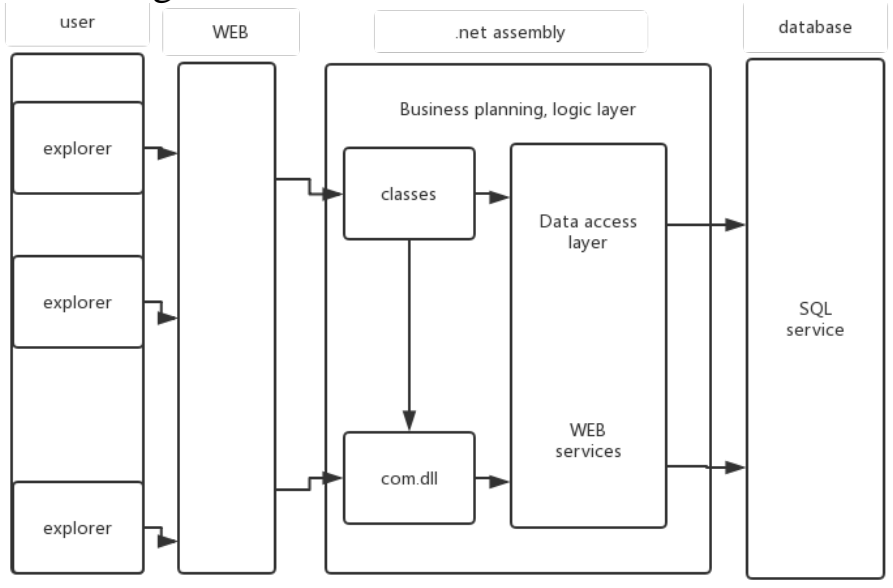

Fig.2 System Three-Layer Structure Diagram

In view of the design of network topology structure, higher vocational colleges mainly explain from the aspects of intelligent grouping and performance analysis system, in which the specific network topology structure is shown in figure 3.

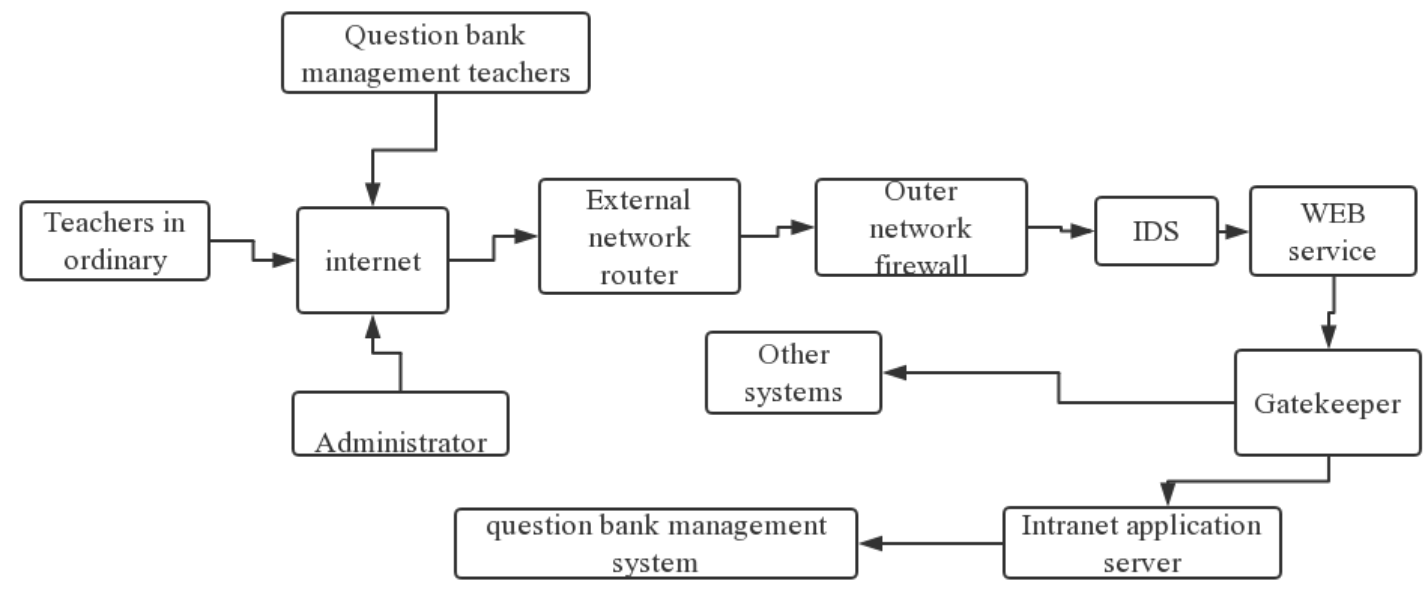

Fig.3 Network Architecture of Question Bank Management System 


\subsection{Detailed Design}

Financial discipline teacher can according to examinee's template is different, to carry on the pertinence difficult easy degree to set, and make the management flow of the question: (1) the question maintenance function: can pass the question of the new, modify, delete and other aspects to update the data. (2) new questions: because the examination is mainly the financial discipline of the teacher, you can according to their daily work in the process of the exercise to expand the question bank. (3) to modify the test questions: since the test was mainly taken by the finance teachers, the test questions with wrong descriptions or wrong answers were modified. (4) to delete the test questions: because the examination is mainly the financial discipline of the teacher, so will be out of the scope or do not have the examination significance of the topic to delete. For the specific flow chart, see figure 4[1-4].

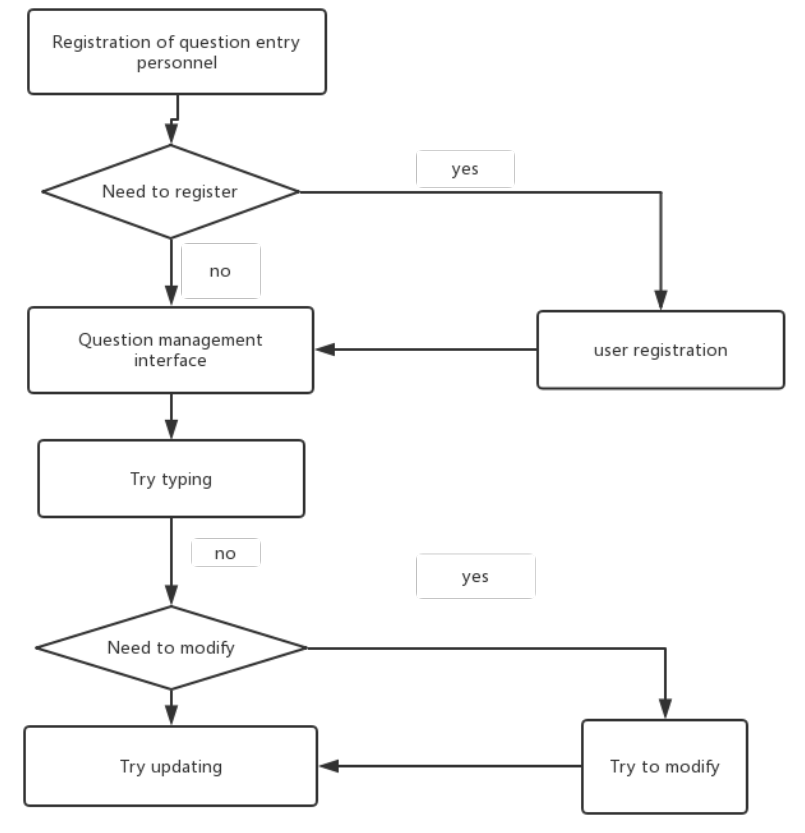

Fig.4 Process Flow Chart for Test Entry

\subsection{Timing Design of Software Functions}

In the selection of algorithm for the examination management system, two algorithms, backtracking algorithm and random algorithm, are firstly analyzed and studied, and the state space is limited. The random algorithm is fast but easy to generate loop condition, which affects the success rate of the test paper. Through the analysis of the data of the two groups of algorithms, it can be known that the current two groups of algorithms cannot meet the requirements of the volume algorithm, so the volume algorithm needs to be updated and optimized. The specific order is shown in figure 5 below [5-8].

\section{System Implementation}

Database concept is mainly from the user's point of view to solve the problem, it has a complete database management system, a good database design structure can lay the foundation through database management, and facilitate the subsequent design.

When the online test ended, financial discipline of teachers can be through the use of smart group volume and test analysis system for automatic marking of mark, the performance of the main process for: students can answer the upload the financial papers submitted to the WEB server, the server will call the answer to the questions and submitted by students, comparing the actual change of examination papers to mark and volume. As for the objective questions, the system generates standard answers through intelligent test paper grouping. In the process of test paper determination, the system can judge whether the answers are correct or not by matching simple strings. As the 
subjective topic involves multiple modes such as artificial intelligence mode, recognition mode and language understanding mode, it still relies on the teacher to judge the paper manually. See figure 6 below for the specific realization model.

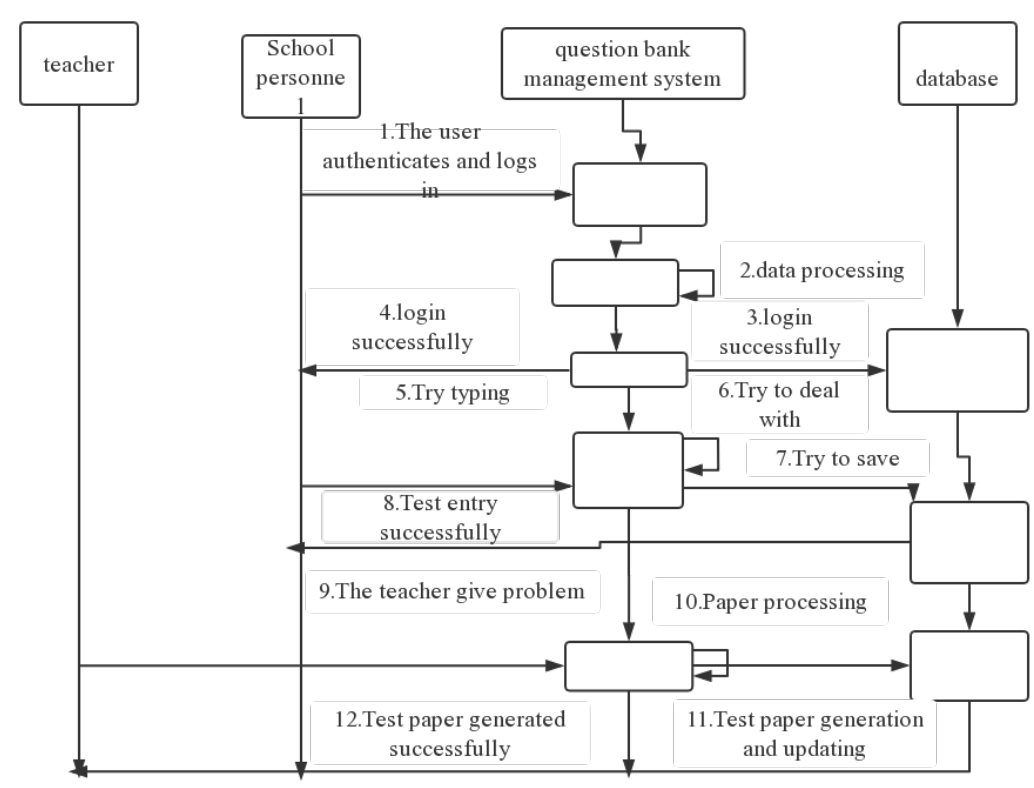

Fig.5 Sequence Diagram of Item Bank Management

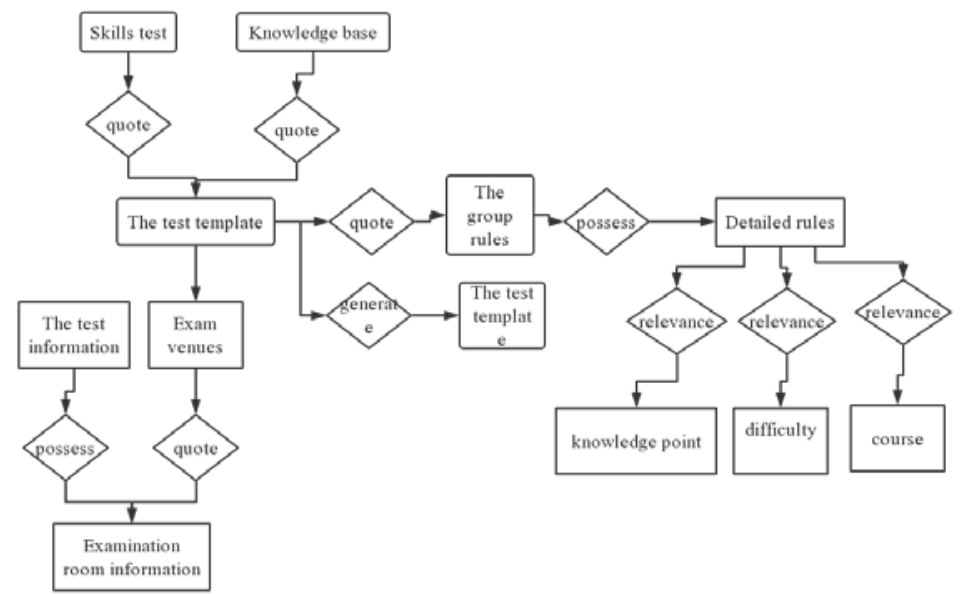

Fig.6 System Implementation Model

\section{Conclusion}

through the higher vocational colleges accounting information of smart group volume and performance analysis system as the research subject, can effective see university informatization is smart group volume and performance analysis system an important part of every college has the characteristics of their respective schools of question bank management system, and according to the actual needs of the universities and colleges, design and develop the most suitable for the colleges and universities the smart group of volume and performance analysis system. The intelligent test paper and performance analysis system in higher vocational colleges has the following characteristics: 1 . With the function of question entry, it can improve the efficiency of manual test paper formation by financial teachers by enhancing the difficulty of the test paper and the knowledge of the test chapter, and classifying the test questions in detail. 2. Financial subject 
teachers in the process of work at ordinary times, through continuous accumulation of different types of questions to update the base test, if the teacher every time in typing in new questions can only separate a test of the input, it will increase the teacher's workload and waste of the teacher's time and experience, through to the base system to increase customers batch import test function, this paper will be through the package in the form of a bulk upload, make the system more human, but also greatly reduce the teacher workload. 3. By implementing manual volume grouping and functional component volume grouping, this will enrich the volume parameters and improve the purpose and pertinence of testing. 4. Through the automatic group volume or artificial volume generated in the form of financial discipline examination paper, financial discipline teacher to repeated use of templates, this will reduce the financial discipline orchestration process of test paper, teacher can make financial focus accounting teaching work, enhances the working efficiency of the financial discipline of teachers, improve the quality of accounting teaching in classroom teaching.

\section{References}

[1] Xiaobing Wang. Design and Implementation of Armoured Soldiers Training Performance Management Information System. South China University of Technology, 2014.

[2] Dolly. The Design and Implementation of Grade Model Examination Management System Based on C\#. Jilin University, 2016.

[3] Hua Xia. Design and Implementation of Data Mining System for Student Performance Analysis. Suzhou University, 2016.

[4] Guolin Chen . Design and Implementation of College Performance Management and Analysis System Based on Decision Tree. Hebei University of Science and Technology, 2016.

[5] Beshan Lati, Gaza. The Design and Implementation of Primary School Performance Management System Based on B/S Model. Xiamen University, 2014.

[6] Qing Sun. Design and Implementation of Monitoring and Analysis System for Teaching Quality in Primary and Middle Schools. Shanghai Jiaotong University, 2013.

[7] Shufen Chen. Design and Implementation of University Teaching Decision Support System Based on Data Mining. University of Electronic Science and Technology, 2012.

[8] Xiaofi Qin. The Design and Implementation of Teaching System Based on WEB. Tianjin University, 2016. 